\title{
Optimal Structure of Computer Numerical Control Grinding Machine Based on Finite Element Method Simulation and Sensor Technology
}

\author{
Kun-Chieh Wang, Chi-Hsin Yang, ${ }^{*}$ Long Wu, and Hao Gao \\ School of Mechanical and Electric Engineering, Sanming University, Sanming, 365004 Fujian Province, China
}

(Received September 14, 2019; accepted February 17, 2020)

Keywords: optimal structure design, CNC machine tools, CAE, finite element, grinding machine, vibration sensor, displacement sensor

For tool machinery design, the type of machine structure plays an important role in its final machining precision. In the marketplace, structure types change very quickly because of different application purposes and customers' requirements. Moreover, to develop new and good machine structures, designers usually pay too much time and money in the work of trial and error. For a quick response to market needs, machine makers should find time-saving and money-saving ways to optimally design and verify their products, and accordingly build a reliable database for further modifying and developing new models. In this study, we propose a hybrid design procedure that uses experience and know-how as initial design bases and, together with an optimal Taguchi-based finite element method (FEM), rapidly and efficiently obtains a highly rigid structure. To verify the simulation results, experiments using displacement sensors are also performed. Specifically, in this study, we chose a long-based computer numerical control (CNC) grinding machine as the target because it is a challenge to attain machining precision owing to its weakness feature of a long and narrow structure. With this proposed methodology, machine designers may efficiently and quickly determine the optimal structure of similar types of CNC machine tools.

\section{Introduction}

To deal with the ever increasing needs of various machining conditions, a large number of computer numerical control (CNC) machines with different functions were continuously fabricated. However, how to speed up the exploration of new machines and assure their quality are still hot issues. From the viewpoints of a machine producer, how to design and produce a reliable machine in time-saving and cost-saving ways is always challenging. To solve this problem, the following basic concepts should be considered first. To design a machine tool with a good structure, there are many factors that affect its precision behavior during machining.

*Corresponding author: e-mail: 20190207@fjsmu.edu.cn https://doi.org/10.18494/SAM.2020.2681 
Among these factors, the original type of structure is especially crucial. In view of the development history of tool machinery for various makers, it is found that most of the machine structures were designed by experience. In the past, it was fine to use experience-based rules to design a simple machine structure with low precision. However, in the highly competitive and ever growing environment today, this experience-based design can no longer provide sufficient knowledge for developing a good-quality machine that frequently requires features such as low weight, multiple functions, and high rigidity, and at the same time, possesses a complex structure. Therefore, for saving development time and money, as well as introducing more knowledge of solid mechanics to cope with the need of a quickly changing market for designing good machines, a rapid and efficient way in conjunction with the previously accumulated knowhow or past knowledge is required.

Thus far, there has been a popular and efficient way to develop or analyze the machine structure, called the "finite element method (FEM)". (1) FEM is a numerical procedure that can be applied to obtain solutions to various problems in engineering. Steady, transient, and linear or nonlinear problems in stress analysis, heat transfer, and fluid flow problems may be analyzed by FEM. The origin of the modern FEM may be traced back to the early 1990s. In the past, many studies using FEM were successfully carried out in many different areas. ${ }^{(2-8)}$ For applications, many general-purpose finite element computer programs were developed, e.g., CATIA, ANSYS, SOLIDWORKS, CREO, INVENTOR, and NASTRAN. Among them, SOLIDWORKS is a comprehensive program that may directly combine the $3 \mathrm{D}$ sketches of a machine structure and perform analysis immediately. Thus far, there have been a large variety of application fields for FEM using these software programs. ${ }^{(6-10)}$

Concerning the investigation of the machine structure of tool machinery, many studies on the stiffness of machine tools using experimental, analytical, or numerical methods have been carried out. ${ }^{(1-13)}$ Specifically, Vivekananda et al. ${ }^{(14)}$ used ANSYS software to obtain the natural frequency of vibration for ultrasonic turning in the machining process. Hong et al. ${ }^{(15)}$ investigated the static structure of the five-axis turning-milling complex CNC machine using the SOLIDWORKS CAE software technique. Wang ${ }^{(16)}$ used FEM with SOLIDWORKS to investigate the static and dynamic responses of the structure of a mill-turn machining center.

In the practical manipulation of designing the structure of a machine, designers usually start with a reference structure that may come from the old database of their company or from competitors. In other words, it can be described in a simple word, "know-how." However, reports that included detailed technical know-how in conjunction with FEM for analyzing the rigidity of machine tools were never seen. It is known that none of the designers dare to design an expensive machine without any reference base. Therefore, to reflect the practical design process and give a complete and fine developing methodology, in this report, we attempt to use the knowledge-based FEM to better (time- and money-saving) design the structure of a machine tool. To identify our proposed methodology, a specific CNC grinding machine used for grinding long rails and guide ways is chosen as the target. This type of machine structure has a long and narrow base, and a complex column-type grinding head. Its structure is weak and usually has a large deformation during machining. Thus, it is a good target to test the result of our study. 
For machine tool structures, two parameters are crucial to their final precision behavior, namely, static rigidity and natural frequency. Static rigidity concerns the magnitude of deformation when applying a static force to the machine, and natural frequency provides the knowledge to understand machine resonance and failure during external periodic stimuli. In the machine structure design, these two parameters interact with each other. Sometimes a structure with good static rigidity would still easily appear damaged owing to resonance or failure during normal cutting operations. Thus, an integrative examination of the effect of the two parameters should be carried out to provide a good overall design guide.

On the other hand, even if one introduces FEM to explore new machine structures in the design process, it still takes much time because too many simulation cases should be accomplished owing to the possible variation of each variable. A famous experimental method called "Taguchi's method" ${ }^{(17)}$ was used to solve this problem, which can largely reduce the number of simulation cases and determine the effect of each controlled variable on the target variable. Moreover, to identify the simulation results, experiments of measuring the static rigidity of a machine via displacement sensors were performed.

As such, in this study, we aim to theoretically use the know-how-based FEM technique together with Taguchi's method and experimentally examine the influence of various machine structures on static rigidity and modal shapes for obtaining an optimal machine structure.

\section{Manipulation Procedure}

The manipulation procedure of our proposed technique includes ten steps, as shown in Fig. 1. First, the target of a challenging long-base grinding machine, which is currently popular in the market, is chosen. All possible related restricted conditions, which include various boundary conditions, material and physical properties of the machine structure, and so on, are indicated. Second, the previously known machine structure information as the basic structure reference (this structure must be tested and its excellent quality confirmed by the market or users) is introduced. Third, a structure prototype is built. Fourth, the governing equations of the static and dynamic behaviors of a solid structure are introduced, and the boundary conditions in mathematic forms are set. Fifth, the dependent and control variables are set, and the signal-to-noise ratio in Taguchi's experiment is defined. Sixth, the mesh structure of the machine under consideration is constructed. Seventh, the FEM calculation using SOLIDWORKS is performed. This includes the following three steps: (1) preprocessing phase (create the solution domain into finite elements, determine the shape function, develop equations for elements, assemble the elements, construct the global stiffness matrix, and apply boundary conditions and loading), (2) solution phase [solve a set of linear or nonlinear algebraic equations to obtain nodal results (displacement, stress, and strain)], and (3) postprocessing phase [obtain other variables (natural frequency and static rigidity)]. Eighth, the static rigidity and natural frequency in every case are analyzed and evaluated. Ninth, the optimal structure for all cases in Taguchi's experiment is determined. Tenth, the obtained optimal structure is identified. 


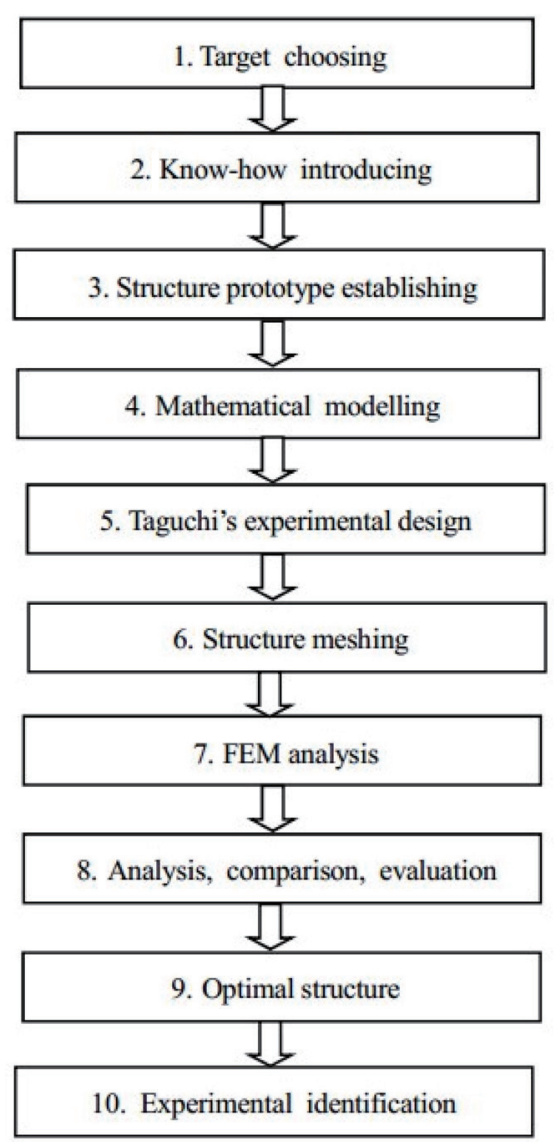

Fig. 1. Manipulation procedure.

\section{Theoretical Bases}

The theories that appeared in our proposed technique include Taguchi's method, static mechanics, vibration theory, and FEM, which are discussed as follows.

\subsection{Taguchi's method}

The technique of laying out the conditions (designs) of experiments involving multiple factors was first proposed in the 1920s. ${ }^{(18)}$ This technique is popularly known as the factorial design of experiments. A full factorial design identifies all possible combinations for a given set of factors. Since most industrial experiments involve a significant number of factors, full factorial design results may involve a large number of experiments. For example, in an experiment involving seven factors, each with two levels, the total number of combinations will be $128\left(2^{7}\right)$. To reduce the number of experiments to a practical level, only a small set from all possibilities is selected. The method of selecting a limited number of experiments that produce the most information is known as a partial factorial experiment. Taguchi constructed a special set of orthogonal arrays (OAs) to lay out his experiments. By combining existing orthogonal 
latin squares in a unique manner, Taguchi prepared a new set of standard OAs, which could be used for a number of experimental situations. He also devised a standard method for the analysis of the results. A single OA may accommodate several experimental situations. Commonly used OAs are available for 2, 3, and 4 levels. The combination of standard experimental design techniques and analysis methods in Taguchi's method produces consistency and reproducibility. Taguchi's method is applied in four steps as follows: (1) brainstorm the quality characteristics and design parameters important to the product/processs, (2) design and conduct the experiments, (3) analyze the results to determine the optimal conditions, and (4) run a confirmatory test using the optimal conditions.

\subsection{Solid mechanics and FEM analysis}

\subsubsection{Static mechanics}

For tool machinery, the governing equation of the structure displacement can be expressed as

$$
[\boldsymbol{K}]\{\boldsymbol{U}\}=\{\boldsymbol{F}\},
$$

or

$$
[\boldsymbol{K}]\{\boldsymbol{U}\}=\left[\boldsymbol{F}^{a}\right]+\left[\boldsymbol{F}^{\prime}\right],
$$

where $[\boldsymbol{K}]$ is the system stiffness matrix, $[\boldsymbol{K}]=\sum_{m=1}^{N}\left[\boldsymbol{K}_{e}\right] ;\{\boldsymbol{U}\}$ is the displacement vector; $N$ is the element number; $\left[\boldsymbol{K}_{e}\right]$ is the element stiffness matrix; $\left[\boldsymbol{F}^{a}\right]$ is the total external force vector; $\left[\boldsymbol{F}^{\prime}\right]$ is the reaction load vector.

In Eq. (2), if sufficient boundary conditions are provided, the displacement at every node $(\{\boldsymbol{U}\})$ may be obtained. If so, Eq. (2) may be rewritten as

$$
\left[\begin{array}{ll}
{\left[\boldsymbol{K}_{n n}\right]} & {\left[\boldsymbol{K}_{n i}\right]} \\
{\left[\boldsymbol{K}_{i n}\right]} & {\left[\boldsymbol{K}_{i i}\right]}
\end{array}\right]\left\{\begin{array}{l}
\left\{\boldsymbol{U}_{n}\right\} \\
\left\{\boldsymbol{U}_{i}\right\}
\end{array}\right\}=\left\{\begin{array}{l}
\left\{\boldsymbol{F}_{n}^{a}\right\} \\
\left\{\boldsymbol{F}_{i}^{a}\right\}
\end{array}\right\}+\left\{\begin{array}{l}
\left\{\boldsymbol{F}_{n}^{r}\right\} \\
\left\{\boldsymbol{F}_{i}^{r}\right\}
\end{array}\right\},
$$

where the subscript " $i$ " means the degree of freedom without displacement restriction. $\left\{\boldsymbol{U}_{i}\right\}$ is known but is not necessarily equal to $\{\boldsymbol{0}\}$. Since the reaction force must be zero when there is no displacement restriction, Eq. (3) may be written as

$$
\left[\begin{array}{ll}
{\left[\boldsymbol{K}_{n n}\right]} & {\left[\boldsymbol{K}_{n i}\right]} \\
{\left[\boldsymbol{K}_{i n}\right]} & {\left[\boldsymbol{K}_{i i}\right]}
\end{array}\right]\left\{\left\{\begin{array}{l}
\left\{\boldsymbol{U}_{n}\right\} \\
\left\{\boldsymbol{U}_{i}\right\}
\end{array}\right\}=\left\{\begin{array}{l}
\left\{\boldsymbol{F}_{n}^{a}\right\} \\
\left\{\boldsymbol{F}_{i}^{a}\right\}
\end{array}\right\}+\left\{\begin{array}{c}
\{\boldsymbol{0}\} \\
\left\{\boldsymbol{F}_{i}^{r}\right\}
\end{array}\right\} .\right.
$$


Solving the upper part of Eq. (4), we may obtain

$$
\left\{\boldsymbol{U}_{n}\right\}=-\left[\boldsymbol{K}_{n n}\right]^{-1}\left[\boldsymbol{K}_{n i}\right]\left\{\boldsymbol{U}_{i}\right\}+\left[\boldsymbol{K}_{n n}\right]^{-1}\left\{\boldsymbol{F}_{n}^{a}\right\} .
$$

The reaction forces $\left\{\boldsymbol{F}_{i}^{a}\right\}$ may be obtained via the upper equation. Solving the lower part of Eq. (4), we may obtain

$$
\left\{\boldsymbol{F}_{i}^{r}\right\}=\left[\boldsymbol{K}_{n i}\right]^{\mathrm{T}}\left\{\boldsymbol{U}_{n}\right\}+\left[\boldsymbol{K}_{i i}\right]\left\{\boldsymbol{U}_{i}\right\}-\left\{\boldsymbol{F}_{i}^{a}\right\} .
$$

The strain energy $\boldsymbol{E}^{S}$ is further obtained as

$$
\boldsymbol{E}^{S}=1 / 2\{\boldsymbol{U}\}^{\mathrm{T}}[\boldsymbol{K}]\{\boldsymbol{U}\}
$$

The relationship between the nodal displacement vector $\{\boldsymbol{u}\}$ and the displacement field is described as

$$
\{\boldsymbol{U}\}=[\boldsymbol{N}]\{\boldsymbol{u}\},
$$

where $[N]$ is the shape function matrix. The strain-displacement relationship is

$$
\{\boldsymbol{\varepsilon}\}=\{\boldsymbol{L}\}\{\boldsymbol{U}\}=\{\boldsymbol{L}\}[\boldsymbol{N}]\{\boldsymbol{u}\},
$$

where $\{\boldsymbol{L}\}$ means the linear differential operator. For a linear structure, the stress vs strain relationship is

$$
\{\boldsymbol{U}\}=[\boldsymbol{N}]\{\boldsymbol{u}\}\{\boldsymbol{\sigma}\}=[\boldsymbol{D}]\{\boldsymbol{\varepsilon}\},
$$

where $[\boldsymbol{D}]$ is the elasticity coefficient matrix.

\subsubsection{Modal analysis}

The modal analysis of a machine structure usually presents its results in the form of natural frequency and mode shape. Three basic assumptions must be made in the modal analysis: (1) the structure is linear, (2) there is no damping effect, and (3) all physical properties under consideration, such as force, displacement, and temperature, are independent of time. In other words, the structure is under free vibration. On the basis of these assumptions, the governing equation of a structure under free vibration may be expressed as

$$
[\boldsymbol{M}]\{\ddot{\boldsymbol{u}}\}+[\boldsymbol{K}]\{\boldsymbol{u}\}=\{\boldsymbol{0}\} .
$$


For a linear structure system, the motion of free vibration is harmonic, i.e.,

$$
\{\boldsymbol{u}\}=\{\boldsymbol{\varphi}\}_{i} \boldsymbol{e}^{j \omega_{i} t}
$$

Here, $[\varphi]$ means the amplitude or mode shape for the $i$ th frequency $\omega_{i}$. Substituting Eq. (12) into Eq. (11), we have

$$
\left[[\boldsymbol{K}]-\omega_{i}^{2}[\boldsymbol{M}]\right]\{\boldsymbol{\varphi}\}_{i}=\{\boldsymbol{0}\}
$$

The above equation is an "eigenvalue problem" in which nontrivial solutions occur under the following condition:

$$
\operatorname{det}\left(\left[[\boldsymbol{K}]-\omega_{i}^{2}[\boldsymbol{M}]\right]\right)=0
$$

From Eq. (14), we may obtain $\mathrm{n}$ numbers of eigenvalues $\left\{\omega_{i}\right\}, \mathrm{i}=1,2, . ., n$ and their corresponding eigenvectors $[\varphi]_{i}, i=1,2, \ldots, n$. The variable $n$ is the number of degrees of freedom of the structure system. For further application, the obtained eigenvectors are usually normalized on the basis of the mass matrix as

$$
\{\boldsymbol{\varphi}\}_{i}^{\mathrm{T}}[\boldsymbol{M}]\{\boldsymbol{\varphi}\}_{i}=1
$$

\section{Results and Discussion}

To obtain a robust structure of a CNC machine, especially the grinding machine with a long travel distance, which appeared in this study, the analysis of structural properties, including static and dynamic rigidities as well as the natural vibration mode, is essential. According to the experience-based initial structure, we may build several different experimental structures and set appropriate boundary conditions via the guide of Taguchi's method. Then, through the FEM calculation of the previously shown governing equations using SOLIDWORKS, we may firstly obtain the corresponding displacement, strain, and stress. Proper suggestions may be provided to modify the weakness points of the machine structure. On the other hand, we may simultaneously analyze the natural vibration modes and forced vibration conditions to understand the dynamic behavior of the interface between parts to avoid resonance and reduce deformation.

In this study, a long-travel CNC grinding machine was chosen as the target. This type of machine features that (1) a long travel ( $y$-direction) may cause a large deviation during machining and (2) the alignment of three-grinding heads is complex and the three-head structure is very heavy. Both factors may easily cause a large vibration error during machining. To solve the above two problems simultaneously, in this study, we provide a good solution. The following are the results of manipulation and related discussions. 


\subsection{Design by experience}

Our reference machine was provided by a specific producer in Taiwan. Presently, this producer is a famous manufacturer of grinding machines, especially for grinding long linear guides (length: greater than $4 \mathrm{~m}$ ). This producer provides a two-head grinding machine (used for grinding a long rail) as our reference, which is presently popular and proved to have a high rigidity in the global market. On the basis of the old two-head structure, we add the third head onto the head ram. The entire machine structure, including the shape of the inner rib of the columns and base, is kept the same, but the three-head assembly and head ram are new designs (Fig. 2). This reference-based three-head case is named case 1. Basically, it is an experiencebased extension of the old two-head structure.

\subsection{Taguchi's experiment}

We are now focusing on how to raise the static and dynamic rigidities of the referencebased machine structure. The machine structure can be divided into four main parts: base, vertical column, cross column, and head ram. The size of each part markedly affects the rigidity of the entire machine. Furthermore, the inner rib shape of each part is also a decision factor affecting the rigidities of each part and the entire machine. As such, the controlled factors can be reasonably chosen as $A-G$, which are defined as $A$ : bed width, $B$ : bed height, $C$ : vertical column length, $D$ : vertical column width, $E$ : inner structure (rib) type, $F$ : cross column height, and $G$ : cross column length. As to the head ram dimension, it is considered to have a fixed size based on the minimal mechanism space requirement. In addition, the grinding-head nose displacement $\left(\delta^{2}\right)$ can be chosen as the dependent variable since it is the most important indexing parameter of precision for a grinding machine. Each controlled factor is set to have two levels. The dependent variable is defined as $y=-10 \log _{10} \delta^{2}$. Then, Taguchi's OA of L8 $\left(2^{7}\right)$ may be established as shown in Table 1.

In the first row, No. 1 represents the initially set structure data originating from the experience (know-how). For all eight experiments, the external forces of $100 \mathrm{Kg}_{f}$ were applied to the nose of the left grinding head in the $Y$ direction, the nose of the right grinding head in
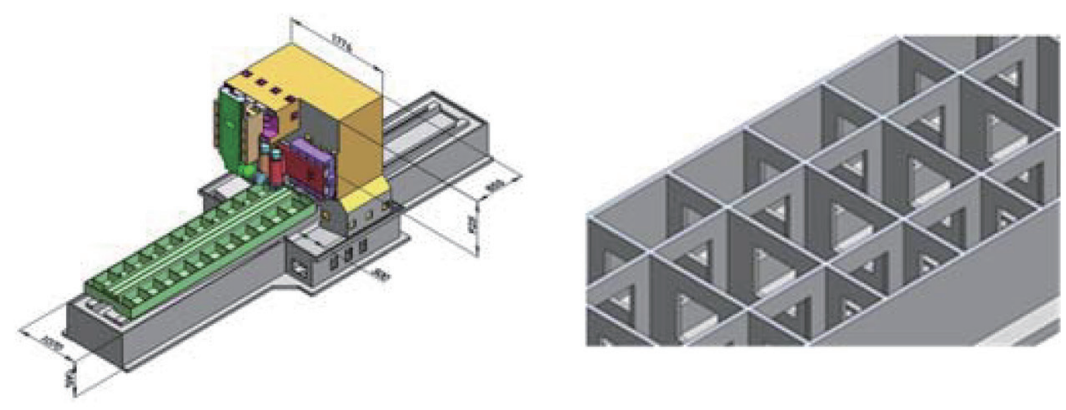

Fig. 2. (Color online) Outer dimensions and inner rib shape of the target machine based on know-how. 
Table 1

\begin{tabular}{|c|c|c|c|c|c|c|c|}
\hline No. & $A$ & $B$ & $C$ & $D$ & $E$ & $F$ & $G$ \\
\hline 1 & 1070 & 590 & 855 & 500 & + & 925 & 1776 \\
\hline 2 & 1070 & 590 & 855 & 600 & * & 1025 & 1926 \\
\hline 3 & 1070 & 690 & 955 & 500 & + & 1025 & 1926 \\
\hline 4 & 1070 & 690 & 955 & 600 & $*$ & 925 & 1776 \\
\hline 5 & 1170 & 590 & 955 & 500 & $*$ & 925 & 1926 \\
\hline 6 & 1170 & 590 & 955 & 600 & + & 1025 & 1776 \\
\hline 7 & 1170 & 690 & 855 & 500 & * & 1025 & 1776 \\
\hline 8 & 1170 & 690 & 855 & 600 & + & 925 & 1926 \\
\hline
\end{tabular}

unit: $\mathrm{mm}$

the $-Y$ direction, and the nose of the upper grinding head in the $Z$ direction. The material of the structures was set as cast iron. Then, the mesh of each structure was established. We performed four grid independence tests with minimal element sizes of 10, 6, 4, and $3 \mathrm{~mm}$. We found that the relative errors of total head displacements are $0.5,0.3,0.0102$, and $0.01017 \%$. Therefore, the element size of $4 \mathrm{~mm}$ was chosen for all cases under consideration. For example, a meshing result of the No. 1 experiment with 125158 nodes and 596491 elements may be obtained.

\subsection{Stress, strain, and displacement}

After completing the meshing process and setting all restricted conditions (shown in Table 1), the stress, strain, and displacement can be calculated for all cases through SOLIDWORKS SIMULATION. For example, Figs. 3 and 4 show the stress and strain distributions of the entire machine. The obtained displacement result of the No. 1 experiment is shown in Fig. 5, which shows a maximum displacement of $14 \mu \mathrm{m}$ occurring at the grinding head nose in the $Z$ direction. The calculated noise results for all cases are shown in Table 2.

Firstly, it is found that no matter what cases are under consideration, the sequence of the magnitude of displacement for parts is always (from large to small) head, head ram, cross column, vertical column, and base. The structures of the head, head ram, and cross column contribute to the major deformation of the entire machine. Secondly, among all the eight cases, case 1 has the minimal noise $y=37.077$. This means that case 1 has the best static structure.

\subsection{Mode shape analysis}

For designing a good structure, both static and dynamic responses should be considered. The dynamic responses include two phases: nature vibration and forced vibration. The mode shape analysis provides a clear insight into the bias of dynamic interaction at the interface between assemblies. Analyzing the type of mode shape under nature vibration and investigating the fragile parts of the machine structure may obtain the modification basis for enhancing the rigidity of the entire machine. 


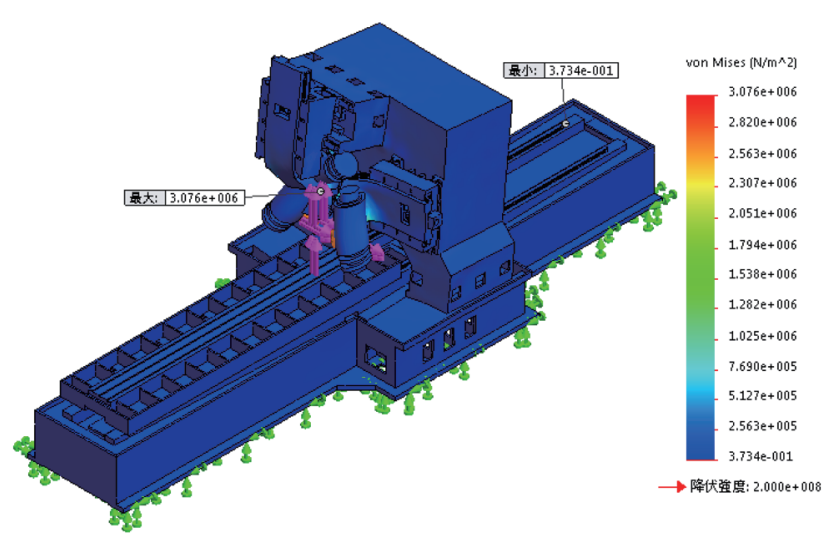

Fig. 3. (Color online) Von Mises stress variation of structure No. 1.

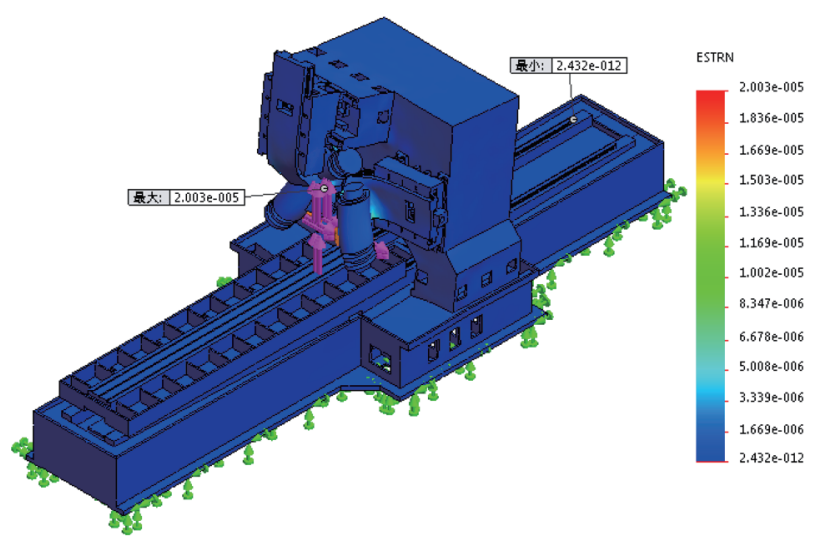

Fig. 4. (Color online) Strain variation of structure No. 1.

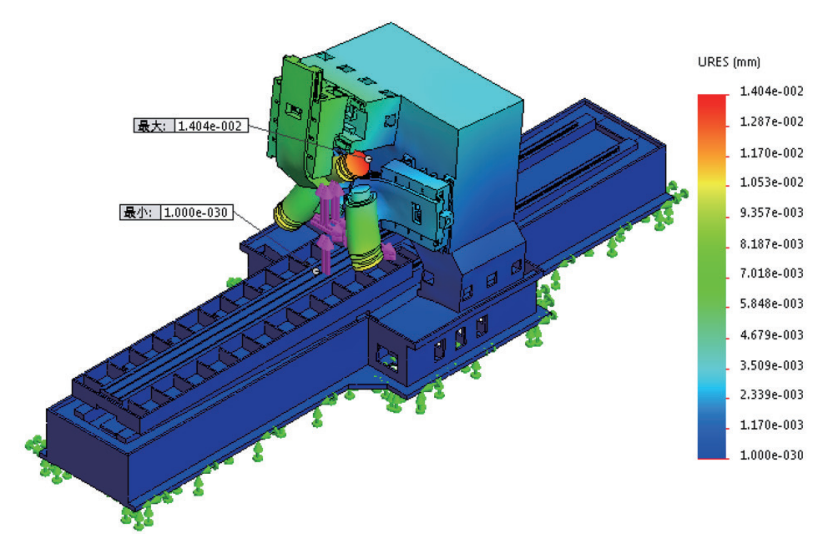

Fig. 5. (Color online) Displacement variation of structure No. 1.

Table 2

Calculation results of noise (Taguchi's experiments).

\begin{tabular}{rcccccrccc}
\hline & $A$ & $B$ & $C$ & $D$ & $E$ & $F$ & $G$ & $\delta$ & $y$ \\
\hline 1 & 1070 & 590 & 855 & 500 & + & 925 & 1776 & .0140 & 37.077 \\
2 & 1070 & 590 & 855 & 600 & $*$ & 1025 & 1926 & .0135 & 37.393 \\
3 & 1070 & 690 & 955 & 500 & + & 1025 & 1926 & .0135 & 37.393 \\
4 & 1070 & 690 & 955 & 600 & $*$ & 925 & 1776 & .0132 & 37.589 \\
5 & 1170 & 590 & 955 & 500 & $*$ & 925 & 1926 & .0132 & 37.589 \\
6 & 1170 & 590 & 955 & 600 & + & 1025 & 1776 & .0135 & 37.393 \\
7 & 1170 & 690 & 855 & 500 & $*$ & 1025 & 1776 & .0138 & 37.202 \\
8 & 1170 & 690 & 855 & 600 & + & 925 & 1926 & .0139 & 37.140 \\
\hline
\end{tabular}

Through FEM calculations, all free vibration mode shapes of eight experiments were obtained. Once these vibration shapes and their corresponding occurrence frequencies are determined, the resonance-free design under normal operation range can be expected. For illustration, the calculated nature frequencies of case 1 are shown in Table 3. It is seen that, 
Table 3

Results of mode shape analysis for case 1 .

\begin{tabular}{|c|c|c|c|c|c|c|c|}
\hline Mode No. & $f(\mathrm{~Hz})$ & Mode No. & $f(\mathrm{~Hz})$ & Mode No. & $f(\mathrm{~Hz})$ & Mode No. & $f(\mathrm{~Hz})$ \\
\hline 1 & 35.9 & 11 & 188.9 & 21 & 282.0 & 31 & 341.4 \\
\hline 2 & 55.5 & 12 & 210.0 & 22 & 286.0 & 32 & 345.0 \\
\hline 3 & 83.0 & 13 & 217.3 & 23 & 290.3 & 33 & 354.1 \\
\hline 4 & 88.6 & 14 & 223.7 & 24 & 293.9 & 34 & 357.7 \\
\hline 5 & 107.6 & 15 & 228.1 & 25 & 297.7 & 35 & 361.2 \\
\hline 6 & 143.0 & 16 & 238.6 & 26 & 311.3 & 36 & 368.4 \\
\hline 7 & 146.0 & 17 & 254.3 & 27 & 317.6 & 37 & 369.2 \\
\hline 8 & 149.1 & 18 & 261.4 & 28 & 323.3 & 38 & 379.2 \\
\hline 9 & 156.3 & 19 & 275.6 & 29 & 331.1 & 39 & 379.8 \\
\hline 10 & 170.2 & 20 & 277.8 & 30 & 334.6 & 40 & 402.1 \\
\hline
\end{tabular}

$f$ : frequency

within the range of $0-100 \mathrm{~Hz}$, there are eight nature frequencies: $35.9,55.5,83.0,88.6,107.6$, 143, 146, and $149.1 \mathrm{~Hz}$. The corresponding first eight mode shapes are shown in Fig. 6. Taking mode shape 1 as an example $(f=35.9 \mathrm{~Hz})$, it is found that the entire column box vibrates backwards and forwards. Moreover, for mode shape $2(f=55.5 \mathrm{~Hz})$, it is found that the entire column box twists. To avoid this vibration phenomenon, the connection interface between the column box and the base should be enhanced so as to change these two natural frequencies.

\subsection{Optimal structure}

Synthesizing the effects of all controlled factors at different levels on the noise in static rigidity experiments, together with considering the natural frequencies, we may obtain the optimal parameters of structure dimensions. Using these parameters, a good grinding machine structure with high rigidity and free of resonance during machining is obtained.

In Table 2, to obtain the effect of every factor (at different levels) on the noise, we now add the noise value at the same level for the same factor. Then, after taking the average of the noise value for each controlled variable at different levels, we may obtain the effect as shown in Table 4. For making the noise as small as possible, the following are observed:

In the 1st column, $y=37.364$ is the smallest value in which it happens at level 1 for factor $A$. In the 2nd column, $y=37.368$ is the smallest value in which it happens at level 1 for factor $B$. In the 3rd column, $y=37.495$ is the smallest value in which it happens at level 2 for factor $C$. In the 4th column, $y=37.340$ is the smallest value in which it happens at level 2 for factor $D$. In the 5th column, $y=37.463$ is the smallest value in which it happens at level 2 for factor $E$. In the 7th column, $y=37.34$ is the smallest value in which it happens at level 2 for factor $G$.

From the above findings, we may expect an optimal structure with dimensions as follows: level 1 of factor $A(1070 \mathrm{~mm})$, level 1 of factor $B(590 \mathrm{~mm})$, level 2 of factor $C(955 \mathrm{~mm})$, level 2 of factor $D(600 \mathrm{~mm})$, level 2 of factor $E$ (*type), level 2 of factor $F(1025 \mathrm{~mm})$, and level 2 of factor $G(1926 \mathrm{~mm})$. 


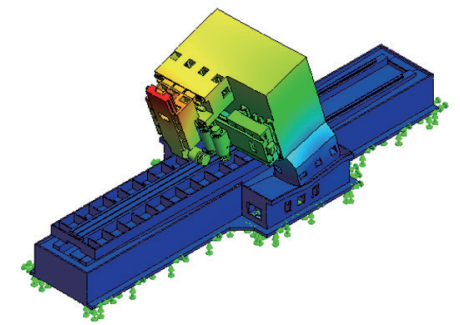

(a)

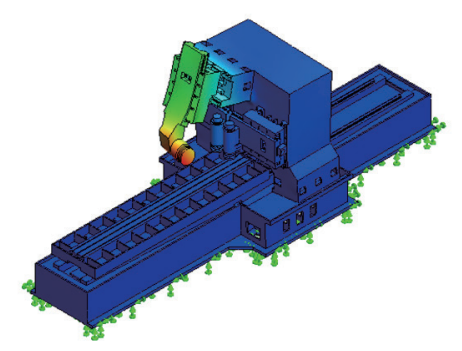

(c)

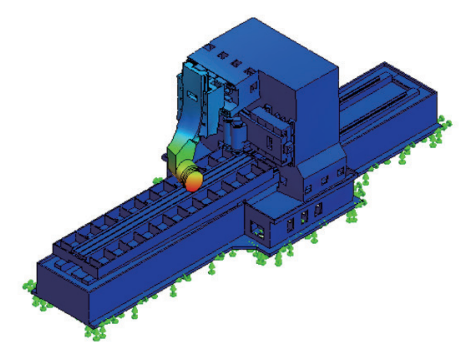

(e)

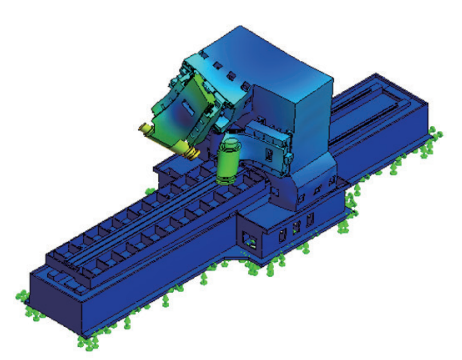

(g)

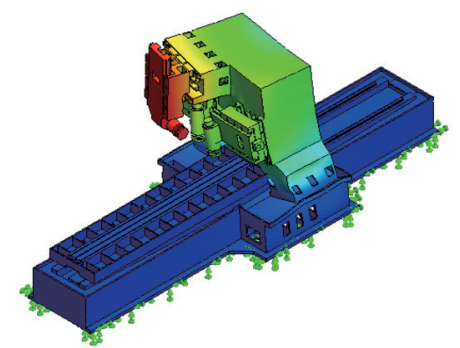

(b)

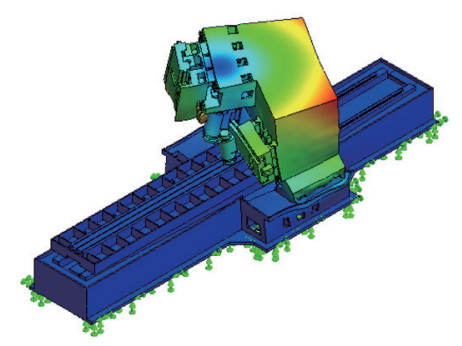

(d)

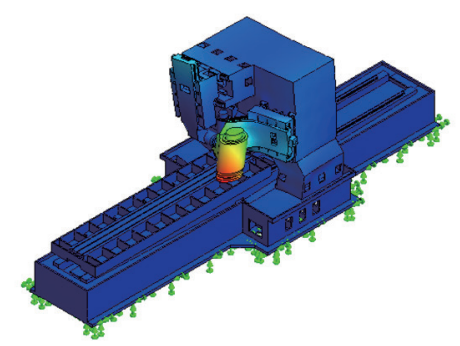

(f)

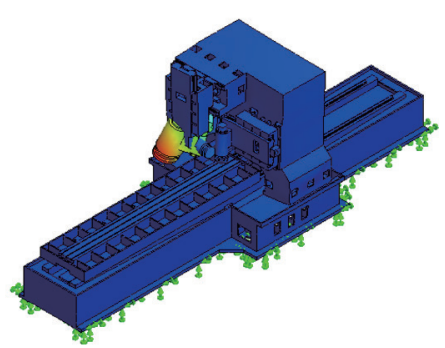

(h)

Fig. 6. (Color online) First eight mode shapes of case 1: (a) mode 1, $f=35.9 \mathrm{~Hz}$; (b) mode 2, $f=55.5 \mathrm{~Hz}$; (c) mode 3 , $f=83.0 \mathrm{~Hz}$; (d) mode 4, $f=88.6 \mathrm{~Hz}$; (e) mode 5, $f=107.6 \mathrm{~Hz}$; (f) mode 6, $f=143.0 \mathrm{~Hz}$; (g) mode 7, $f=146.0 \mathrm{~Hz}$; (h) mode $8, f=149.1 \mathrm{~Hz}$.

Table 4

Effects of controlled factors on noise $(y)$.

\begin{tabular}{lccccccc}
\hline Factors & $A$ & $B$ & $C$ & $D$ & $E$ & $F$ & $G$ \\
\hline Level 1 & 37.364 & 37.368 & 37.167 & 37.322 & 37.199 & 37.304 & 37.318 \\
Level 2 & 37.298 & 37.294 & 37.495 & 37.340 & 37.463 & 37.358 & 37.344 \\
Effect & 0.067 & 0.074 & -0.330 & -0.018 & -0.265 & -0.054 & -0.027 \\
\hline
\end{tabular}




\subsection{Theoretical and experimental verifications}

To verify the proposed optimal structure, we now calculate its static rigidity, natural frequency as well as its corresponding mode shape. This case is named No. 9 with related dimension parameters as shown in Table 5. All restricted conditions are kept the same as the previous eight cases. First, the calculated displacement distribution is shown in Fig. 7. The maximum displacement is $0.0131 \mathrm{~mm}$, which is $7.2 \%$ smaller than that of case $1(0.01404 \mathrm{~mm}$, the best one with the highest static rigidity among the eight cases). Second, the partially obtained natural vibration responses (the first 38 natural frequencies, under $400 \mathrm{~Hz}$ ) are shown in Table 6 and their corresponding first eight mode shapes are shown in Fig. 8. It is found that the first mode shape occurs at $40 \mathrm{~Hz}$, with a forward-and-backward vibration condition, which

Table 5

Dimensions and rib shape of the optimal machine structure.

\begin{tabular}{lccccccc}
\hline No. & $A$ & $B$ & $C$ & $D$ & $E$ & $F$ & $G$ \\
\hline 9 & 1070 & 590 & 955 & 600 & $*$ & 1025 & 1926 \\
\hline unit: $\mathrm{mm}$ & & & & & & &
\end{tabular}

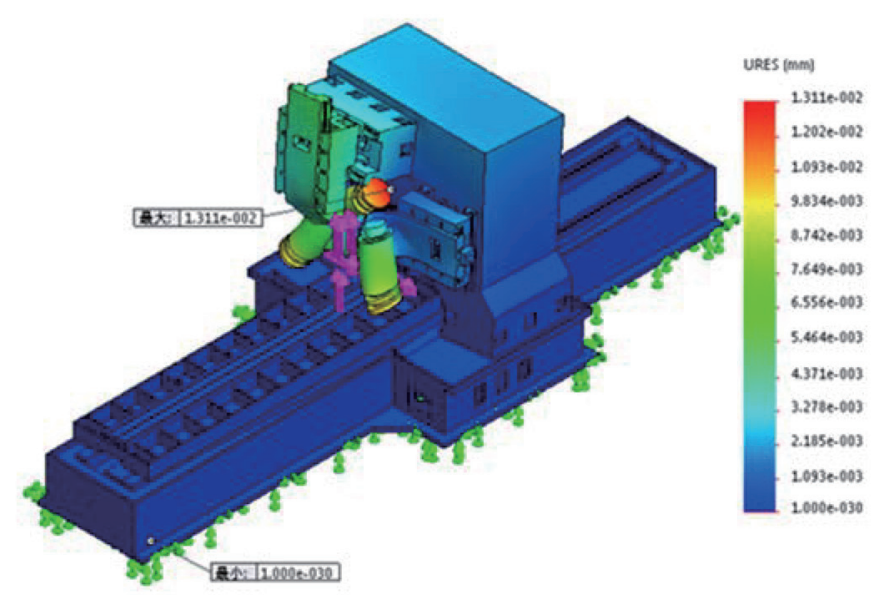

Fig. 7. (Color online) Displacement distribution of case 9.

Table 6

Mode shape analysis (case 9).

\begin{tabular}{|c|c|c|c|c|c|c|c|}
\hline No. & $f(\mathrm{~Hz})$ & No. & $f(\mathrm{~Hz})$ & No. & $f(\mathrm{~Hz})$ & No. & $f(\mathrm{~Hz})$ \\
\hline 1 & 40.0 & 11 & 206.6 & 21 & 282.5 & 31 & 338.5 \\
\hline 2 & 60.5 & 12 & 214.0 & 22 & 289.2 & 32 & 347.5 \\
\hline 3 & 87.0 & 13 & 222.1 & 23 & 291.3 & 33 & 353.1 \\
\hline 4 & 91.0 & 14 & 231.4 & 24 & 294.5 & 34 & 362.3 \\
\hline 5 & 108.1 & 15 & 236.6 & 25 & 298.7 & 35 & 366.7 \\
\hline 6 & 145.8 & 16 & 249.4 & 26 & 305.2 & 36 & 375.1 \\
\hline 7 & 146.8 & 17 & 252.3 & 27 & 309.3 & 37 & 382.7 \\
\hline 8 & 150.6 & 18 & 262.7 & 28 & 311.5 & 38 & 397.0 \\
\hline 9 & 154.3 & 19 & 266.2 & 29 & 320.6 & & \\
\hline 10 & 170.5 & 20 & 272.2 & 30 & 336.1 & & \\
\hline
\end{tabular}




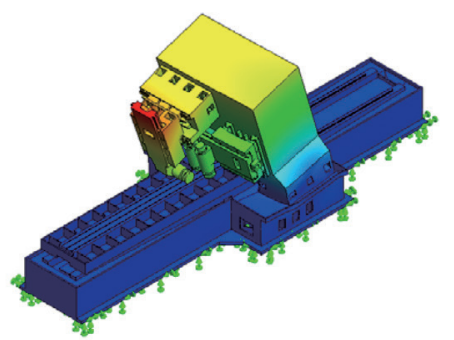

(a)

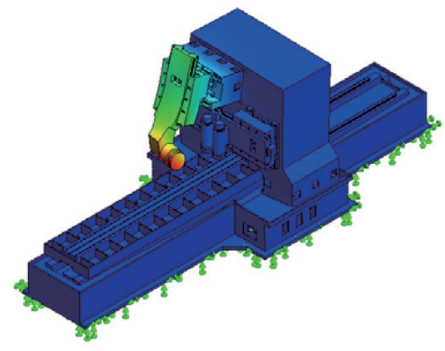

(c)

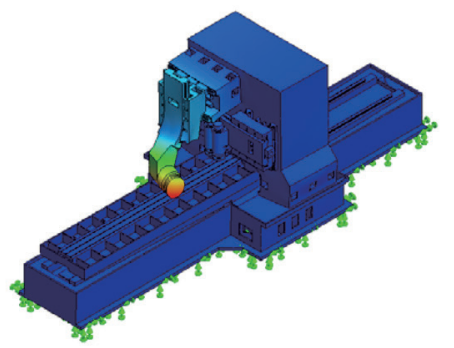

(e)

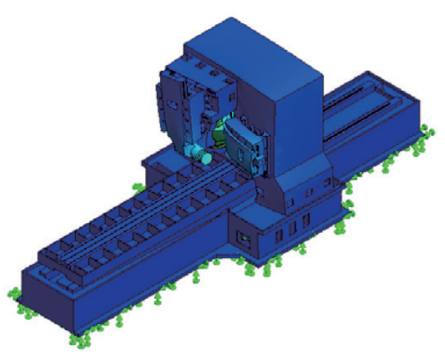

(g)

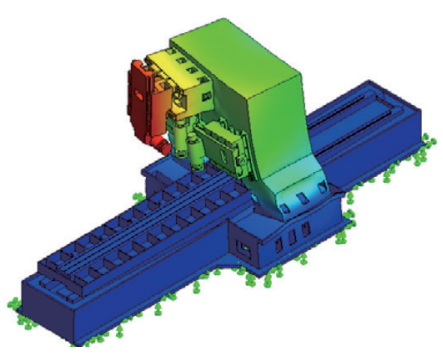

(b)

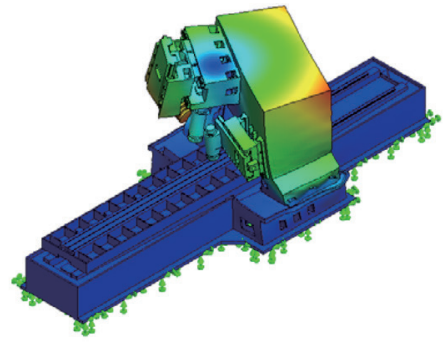

(d)

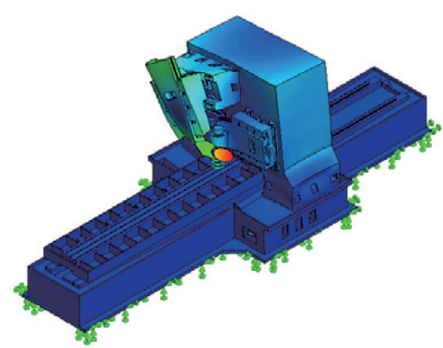

(f)

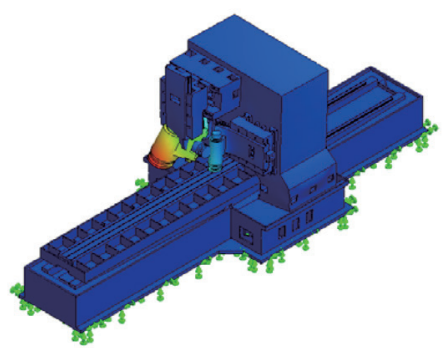

(h)

Fig. 8. (Color online) Mode shape of case 9 (first eight modes): (a) mode 1, $f=40.0 \mathrm{~Hz}$; (b) mode 2, $f=60.5 \mathrm{~Hz}$; (c) mode 3, $f=83.0 \mathrm{~Hz}$; (d) mode 4, $f=91.0 \mathrm{~Hz}$; (e) mode 5, $f=108.1 \mathrm{~Hz}$; (f) mode 6, $f=145.8 \mathrm{~Hz}$; (g) mode 7 , $f=146.8 \mathrm{~Hz}$; (h) mode $8, f=150.6 \mathrm{~Hz}$.

is about $10 \%$ higher than that of case 1 . In the frequently used cutting range of $0-3000 \mathrm{rpm}$ $(0-50 \mathrm{~Hz})$, this specific frequency $(40 \mathrm{~Hz})$ would possibly induce resonance. The machine designer should be aware and make some adjustments (e.g., spindle not operating at this speed) to avoid or transfer this condition. 
On the other hand, a displacement measurement experiment was performed to examine the static rigidity of the obtained optimal structure of the CNC grinding machine (case 9). A total of six strain gauge sensors were stamped on the bottom surface of each grinding spindle to measure the spindle displacements that resulted from applying $100 \mathrm{kgf}$ forces in the $Y,-Y$, and $Z$ directions via a load cell at the bottom of the left, right, and upper grinding heads. Among them, two strain gauge sensors were on the upper grinding spindle, two on the right grinding spindle, and the other two on the left grinding spindle. The measured maximum deformation is $0.01426 \mathrm{~mm}$, occurring at the bottom of the upper grinding spindle, which corresponds to a relative error of $8.85 \%$ relative to the simulation results.

\section{Conclusions}

In this study, we used the know-how-based computer-aided engineering technique together with Taiguchi's OA to efficiently explore the structure of a CNC grinding machine. Two critical parameters of the machine structure, namely, static rigidity and vibration mode shape, were investigated simultaneously to obtain an optimal structure that ensures a high-precision behavior during machining. Results show that (1) the know-how-based structure prototype can give an efficient guide to develop a new structure model. (2) Taguchi's experiment of OA can largely reduce the number of experiments and determine the effects of controlled factors (structure parameters) on the target variable (grinding-head nose displacement). (3) The FEM technique, with the least price, may be used to obtain a detailed insight into the machine structure and find out how to design a good machine structure. (4) Combining the above three techniques results in a quick and efficient way of exploring a good machine structure with high rigidity. Both theoretical simulations and displacement-sensor-based experiments were performed to verify the obtained optimal structure, and the comparison results are satisfactory.

\section{Acknowledgments}

The authors acknowledge the financial support from the Training Program of Outstanding Discipline and Specialty Leaders of Fujian Province Universities and Institutions, the China postdoctoral fund (2013M541851), and the Operational Funding of the Advanced Talents for Scientific Research (19YG05) supported by Sanming University. Technical supports from the following institutions are also acknowledged: Fujian Public Service Platform for miniEREV Power System, Fujian Provincial University Engineering Research Center for Modern Mechanical Design and Manufacturing Technology, Fujian Provincial Collaborative Innovation Center for Green Casting, Forging and Advanced Manufacturing, and Fujian Provincial Engineering Research Center for Casting and Forging Parts.

\section{References}

1 S. Moaveni: Finite Element Analysis, Theory, and Application with ANSYS, Global Edition, 4/E (Pearson, Edinburgh Gate, 2014) p. 21.

2 R. Mahdavinejad: Int. J. Mach. Tools Manuf. 45 (2005) 753. https://doi.org/10.1016/j.ijmachtools.2004.11.017 
3 J. J. Wu: Int. J. Mech. Sci. 46 (2004) 1245. 2004. https://doi.org/10.1016/j.ijmecsci.2004.07.002

4 S. Yang: Int. J. Mach. Tool Des. and Res. 21 (1981) 23. https://doi.org/10.1016/0020-7357(81)90011-1

5 D. T. Y. Huang and J. J. Lee: Int. J. Mach. Tools Manuf. 41 (2001) 1149. https://doi.org/10.1016/s08906955(01)00012-8

6 Z. Liu: Multiphysics in Porous Materials: Finite Element Method (Springer, Switzerland AG, 2018) p. 397.

7 S. H. Chen: Computational Geomechanics and Hydraulic Structures: Fundamentals of the Finite Element Method (Springer, Singapore, 2019) p. 241.

8 L. Chu, J. Shi, and E. S. Cursi: Nanomaterials 8 (2018) 489. https://doi.org/10.3390/nano8070489

9 J. Majerik and J. Jambo: Procedia Eng. 100 (2015) 450. https://doi.org/10.1016/j.proeng.2015.01.390

10 E. Wagner: Procedia Eng. 19 (2016) 34. 2015. https://doi.org/10.1016/j.protcy.2015.02.006

11 D. Kano, T. Lorenzer, S. Weikert, and K. Wegener: Precis. Eng. 34 (2010) 399. https://doi.org/10.1016/ j.precisioneng.2009.09.003

12 J. J. Wu: Meas. 39 (2006) 740. https://doi.org/10.1016/j.measurement.2006.03.002

13 Y. Kang, Y. P. Chang, J. W. Tsai, S. C. Cheng, and L.K. Yang: Finite Elem. Anal. Des. 37 (2001) 485. https:// doi.org/10.1016/S0168-874X(00)00049-4

14 K. Vivekananda, G. N. Arka, and S. K. Sahoo: Procedia Mater. Sci. 6 (2014) 1906. https://doi.org/10.1016/ j.mspro.2014.07.223

15 C. C. Hong, C. L. Chang, and C. Y. Lin: Eng. Sci. and Tech. 19 (2016) 1971. https://doi.org/10.1016/ j.jestch.2016.07.013

16 K. C. Wang: Eng. Comp. 31 (2014) 1761. https://doi.org/10.1108/ec-11-2012-0296

17 H. Unal, F. Ficici, A. Mimaroglu, A. Demirkol, and A. Kırdar: J. Braz. Soc. Mech. Sci. Eng. 38 (2016) 2097. http://doi.org/10.1007/s40430-015-0398-1

18 W. H. Yang and Y. S. Tarng: J. Mater. Process. Technol. 84 (1998) 122. https://doi.org/10.1016/S09240136(98)00079-X 